\title{
SPLEEN PRESERVING DISTAL PANCREATECTOMY FOR SOLID PSEUDO-PAPILLARY TUMOUR OF THE BODY AND TAIL OF THE PANCREAS
}

\author{
Banagala A S K ${ }^{1}$, Nanadasena A C N², Wasalaarachchi K C K ${ }^{3}$, Ariyaratne M A Y ${ }^{4}$, Liyanage A S ${ }^{5}$ \\ ${ }^{1}$ Consultant General Surgeon, Colombo South Teaching Hospital, Kalubowila. ${ }^{2}$ Consultant \\ Urological Surgeon, Colombo North Teaching Hospital, Ragama. ${ }^{3}$ Consultant Histopathologist, \\ Colombo South Teaching Hospital, Kalubowila. ${ }^{4}$ Consultant Clinical Oncologist, National Cancer \\ Institute, Maharagama. ${ }^{4}$ Registrar in General Surgery, Colombo South Teaching Hospital, Kalubowila.
}

A S K Banagala MS, FRCSI

Consultant General Surgeon, Colombo South Teaching Hospital, Kalubowila.

A C N Nandasena MS

Consultant Urological Surgeon,

Colombo North Teaching Hospital, Ragama.

K C K Wasalaarachchi MD

Consultant Histopathologist,

Colombo South Teaching Hospital, Kalubowila.

MAY Ariyaratne MD

Consultant Clinical Oncologist,

National Cancer Institute, Maharagama.

A S Liyanage MBBS

Registrar in General Surgery,

Colombo South Teaching Hospital, Kalubowila.

Corresponding author:

A S K Banagala

Colombo South Teaching Hospital,

Kalubowila, Dehiwala, Sri Lanka.

\section{Introduction}

A fourteen year old girl presented to us with persistent left hypochondrial and left loin pain for several months, associated with loss of weight and dyspeptic symptoms. Physical examination did not reveal any significant findings. Urinalysis, full blood count, abdominal and chest radiographs were normal. Erythrocyte sedimentation rate was $72 \mathrm{~mm}$ for the first hour. Abdominal ultrasound scan too, was unremarkable.

As the symptoms were considered to be significant by the clinicians, an abdominal CT scan was performed which showed a tumour involving distal body and tail of the pancreas with enlarged peripancreatic lymph nodes. Ultrasound guided biopsy of the tumour was attempted, but was not successful. The patient and her parents were informed of the findings. Consent was obtained from the parents for distal pancreatectomy, including splenectomy. However, we also explained that, if technically feasible, spleen would be saved. Pneumococcal vaccine and haemophilus influenzae vaccine were administered three weeks prior to surgery. Under general anaesthesia a curved transverse incision midway between umbilicus and xiphoid, and convex upwards, was made and greater omentum was divided outside the epiploic vessels. All the short gastric vessels were divided between haemostats and ligated. Splenic flexure of the colon was mobilized to facilitate access to the pancreas. Dissection of the pancreatic tail off the spleen and the splenic artery and vein was performed meticulously. Most of the arterial branches and venous tributaries were easily controlled with electrocautery. A few vessels needed ligation with $3 / 0$ polygalactin suture. The pancreas was divided transversely between the neck and the body. The cut end of the pancreatic duct was ligated. The distal stump was oversewn with $2 / 0$ polygalactin suture. The enlarged lymph nodes along the splenic artery and the left gastric artery were carefully dissected. A wide bore drain was placed next to the pancreatic stump and routine closure was performed. The drain was removed on the fifth day and the patient was discharged. She had one episode of mild acute pancreatitis two weeks later, but remains otherwise healthy up to date. A repeat CT scan performed six months later did not reveal any local recurrence. 


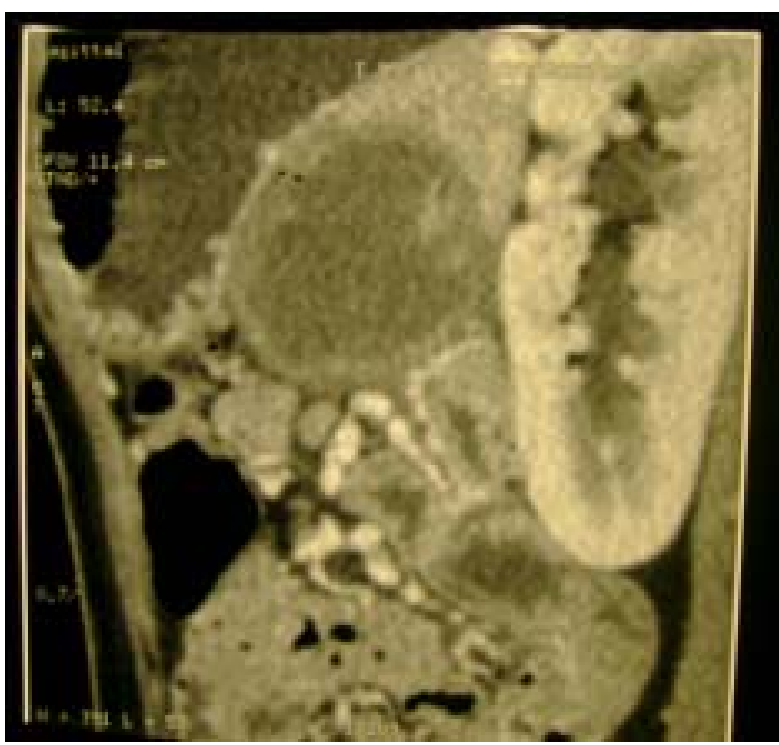

Figure 1. Sagital reconstruction of the CT abdomen, showing the pancreatic tumour lying anterior to the left kidney.

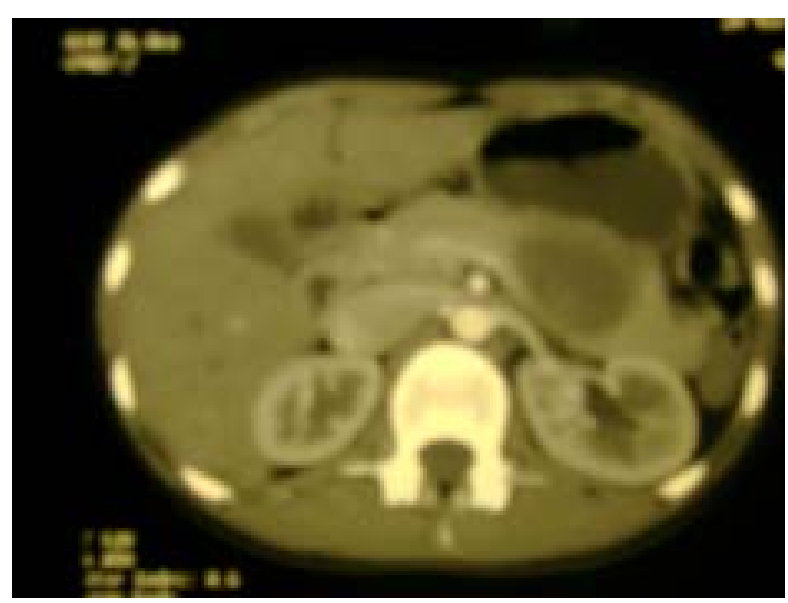

Figure 2. Non contrast CT abdomen showing the pancreatic tumour lying anterior to the left kidney.

\section{Discussion}

Solid pseudopapillary tumour of the pancreas is rare and accounts for $1-2 \%$ of exocrine pancreatic tumours (1). These tumours are indolent and only $15 \%$ of them would produce metastases. Even when metastases do occur, they are often amenable to surgical resection and patients rarely die as a direct result of this neoplasm (2). Pathological features that indicate the degree of aggressiveness are, venous invasion, degree of nuclear atypia, mitotic rate, and prominence of necrobiotic cell nests (3). Solid pseudopapillary tumours are typically positive for vimentin, neuron specific enolase(NSE), alpha 1 antitrypsin, alpha 1 antichymotrypsin, and are negative for chromogranin, epithelial membrane antigen, and cytokeratin (1). Aggressive attempts at complete resection are warranted on the basis that recurrence has not been observed after complete excision of the local disesase (2).

Splenic preservation following distal pancreatectomy is safe, for benign or low grade malignant disease, and is associated with a reduction in perioperative infective complications, severe complications and length of hospital stay compared with conventional distal pancreatectomy with splenectomy (4). There are two technical approaches. The first preserves the short gastric vessels and the splenic vessels are ligated at the hilum of the spleen (5). In the second, the splenic vessels are preserved by meticulous dissection with ligation of tributaries/branches. Long term follow-up of patients has shown that such preservation of splenic vessels does not compromise the oncological clearance of the local disease (6).

\section{References}

1. Washington K. Solid papillary tumour of the pancreas: challenges presented by an unusual pancreatic neoplasm. Ann Surg Oncol 2002; 9: 3-4.

2. Martin RCG, Klimstra DS, Brennan MF, Conlon KC. Solid pseudopapillary tumour of the pancreas: a surgical enigma? Ann Surg Oncol 2002; 9: 35-40.

3. Nishihara K, Nagoshi M, Tsuneyoshi M. Papillary cystic tumours of the pancreas. Asessment of their malignant potential. Cancer 1993; 71: 82-92.

4. Shoup M, Brennan MF, McWhite K, Leung DHY, Klismstra D, Conlon KC. Value of splenic preservation with distal pancreatectomy. Arch Surg 2002; 137: 164-8.

5. Warshaw AL. Conservation of Spleen with distal pancreatectomy. Arch Surg 1988; 123: 550-3.

6. Cooper MJ, Williamson RCN. Conservative pancreatectomy. Br J Surg 1985; 72: 801-3. 